The objective of this study is to assess the prevalence of musculoskeletal disorders (MSDs) among Medical Secretaries (MS) and their impact on their health-related quality of life (HRQL).

Methods A cross-sectional multicentric exhaustive study was conducted among medical secretaries working in three public hospitals. The survey included a self-administered questionnaire related to individual and professional characteristics, a French abbreviated version of the Karasek's Job Content Questionnaire, a French validated version of Nordic Questionnaire, a French validated version of the SF12 and NHP scores. The study also included objective validated clinical maneuvers. Determinants of MSDs and their impact on HRQoL were evaluated through univariate and multivariate analysis.

Results A total of 72 office female workers were included with a response rate of $81.8 \%$. The mean age was $43.75 \pm$ 8.9 years. The body mass index (BMI) was higher among workers over the age of 45 years. According to Karasek's model, 54 subjects (75\%) were in 'Job strain' situation. During the last 12 months, back complaints were reported by $69.4 \%$ and neck complains were reported by $79.2 \%$ of workers. An impaired physical HRQoL (PCS <50,11) was identified in $72.2 \%$ of subjects. An altered mental HRQoL (MCS $<47.96)$ was identified in $76.4 \%$ of workers. In the multivariate analysis, BMI was found to be a risk factor for MSDs of the back (OR: 1.2), whereas time spent in front of the computer screen was found to be a risk factor for MSDs of the neck (OR: 2.8). MSD of the back was an independent predictive factor for a more altered physical HRQoL (OR: 7.45), whereas MSD of the knees was an independent predictive factor for more altered mental HRQoL (OR: 1.87).

Conclusion The prevalence of MSDs among female office workers is high and have a negative impact on both physical and mental HRQoL.

\section{P-374 LIFE AND DEATH AT WORK: A TUNISIAN PROSPECTIVE STUDY}

'Amira Omrane, Mohamed Amine Mesrati, Lamia Bouzgarrou, Taoufik Khalfallah, Abir Aissoui. ${ }^{1}$ Faculty of Medicine of Monastir, Tunisia

\subsection{6/OEM-2021-EPI.296}

Introduction Death at work remains a frequent pressing public health issue despite undeniable progress in occupational health and safety. This study aims to assess cases of deaths at the workplace reported in the Forensic Medicine Department of a public hospital in the central region of Tunisia.

Patient and Methods A prospective exhaustive study was conducted in the Forensic Medicine Department of a public hospital located in the central region of Tunisia (Mahdia). This study concerned death occurring in the workplace for which a forensic investigation was carried out during a period of four years from January 2016 to December 2019.

Results Of the 728 deaths reported to the Forensic Medicine Department during the study period, only thirty-five deaths occurred in the workplace with an incidence of $4.8 \%$. The deceased were mostly men (sex ratio $=4$ ) and mean aged 48 years. Victims were blue-collar workers in $97 \%$ of cases, fishermen in $34 \%$ of cases, security guards in $14 \%$ of cases, construction workers in $12 \%$ and finally agricultural labourers in $2 \%$ of cases. The mechanism of death mostly reported was drowning (34\%) followed by cardiac accidents (23\%) and trauma (16\%). This study revealed a case of suicide in the workplace and two cases of white autopsy.

Conclusion Deaths in the workplace fall into two broad categories: deaths due to workplace injuries or fatal work- related accidents including motor vehicle accidents, machinery-related events, homicides, falls, and electrocution, and deaths due to chronic diseases such as ischemic accidents, aortic dissection. This study has highlighted the contribution of early autopsy in revealing the cause of deaths occurring in the workplace and the need to implement preventive measures to minimize workplace-related preventable deaths.

\section{P-375 OCCUPATIONAL FUTURE OF HEALTHCARE WORKERS WITH OCCUPATIONAL DERMATITIS.}

'Amira Omrane, Harrathi Chayma, Asma Kheder, Maher Maoua, Lamia Bouzgarrou, Taoufik Khalfallah, Najib Mrizak, Mohamed Akrout, Adnene Hanchi, Hichem Belhadj Ali. ${ }^{1}$ Faculty of Medicine of Monastir, Tunisia

\subsection{6/OEM-2021-EPI.297}

Aim To identify factors influencing the occupational future of healthcare workers suffering from occupational dermatitis.

Patients and Methods This was a multicenter cross-sectional study of healthcare workers suffering from occupational dermatosis in the four public hospitals in the central region of Tunisian. A synoptic sheet related to socio-professional and administrative data was completed. A self-questionnaire relative to medical data and occupational future was completed during a direct interview.

Results The study involved 40 healthcare workers, only 37 workers were included in the study. They were predominantly female (73\%) and mean aged $44.7 \pm 9.4$ years. A request of reclassification was introduced in 19 cases (51\%), workstation adaptation in 14 cases (38\%) and allergen eviction in 20 cases (54\%). A mutation was reported in two patients $(5.4 \%)$ and three workers retired (8.1\%). A statistically significant association was found between the request of reclassification, a history of allergic manifestations ( $\mathrm{p}=$ $0.003)$ and the allergic agent $(p=0.014)$. Workstation layout was significantly associated with a history of allergic manifestations $(\mathrm{p}=0.039)$, the hand palm location ( $\mathrm{p}=$ 0.04 ), and eviction measures. After multiple binary logistic regression, the request of reclassification was significantly correlated with a history of allergic manifestations ( $\mathrm{p}=$ $0.008)$, a sensitization to nickel sulfate $(p=0.009)$ and the fingers location $(\mathrm{p}=0.038)$. The change of workstation was significantly correlated with a history of allergic manifestations $(\mathrm{p}=0.026)$.

Conclusion The occupational future of healthcare workers suffering from occupational dermatitis depends on a history of atopy (especially allergic rhinitis) and sensitization to allergens (thiuram mix).

\section{P-376 ORIGIN, PREVALENCE AND DETERMINANTS OF VIOLENCE IN PUBLIC HOSPITALS IN TUNISIA.}

${ }^{1}$ Amira Omrane, Olfa Jlassi, Imen Mlouki, Taoufik Khalfallah, Lamia Bouzgarrou, Sana Mhamdi, Myriam Ouerchefani. 'Faculty of Medicine of Monastir, Tunisia

10.1136/OEM-2021-EPI.298 